\title{
CAPÍtulo VII \\ EL ESTUDIO DE LA POLÍTICA EN LA FORMACIÓN PROFESIONAL
}

\author{
Eduardo Gómez ${ }^{1}$ \\ Diana Marcela Pinto ${ }^{2}$
}

\section{Introducción}

En los diversos espacios cotidianos el ser humano se ve inmerso en múltiples interacciones que hacen que se relacione con otros, y por ende, que se enfrente con variadas maneras de ser, sentir y actuar en el mundo. Tal situación conlleva a buscar mecanismos que le permitan mantener una sana convivencia y la posibilidad de configurar espacios para el bien común. Estas dinámicas resaltan el ejercicio político como parte vital humana.

1 Licenciado en Filosofía y Letras, Universidad Santo Tomás de Bogotá. Especialista en Filosofía de la Ciencia, Universidad del Bosque. Magíster en Estudios Políticos, Pontificia Universidad Javeriana de Bogotá

2 Licenciada en Filosofía, Corporación Universitaria Minuto De Dios - Uniminuto. Especialista en Educación, Cultura y Política, Universidad Nacional Abierta y a Distancia - UNAD. Magíster en Desarrollo Educativo y Social, Universidad Pedagógica Nacional — U.P.N. 
Ya desde la antigüedad la política, como forma de vida, era considerada como una dimensión social y comunitaria de la inserción orgánica de la comunidad (polis), y por ello la participación activa y continuada estaba presente en sus ideales acerca de la naturaleza y funciones del Estado, de las instituciones democráticas y las formas de control social. Esta conciencia política como conciencia ciudadana y democrática ha ido desarrollándose en formas diferentes a través de la historia y de los diversos intentos de modelos de sociedad que quieren hacer de la participación el eje de la actividad política.

La importancia que reviste la política como dimensión humana y social vital, lleva a la necesidad de promover la formación política especialmente en la juventud, que hoy se encuentra con la problemática de la indiferencia, la apatía y el proceso creciente de la despolitización. Así mismo, la mentalidad de hoy influenciada por las ideas políticas actuales rechaza cualquier forma de proyecto sociopolítico y de compromiso radical. Los ideales de justicia y solidaridad se ven cuestionados; se privilegian el presente total y absoluto, en función de la realización del individuo y la búsqueda del confort y el placer: es el glorificar al individuo con su egoísmo.

Es por ello que en la educación superior es pertinente que exista una asignatura de política que permita concientizar, informar y formar en los más influyentes ideales y sistemas políticos y autores que han reflexionado sobre la construcción de una sociedad democrática y plural. La clase no debe orientarse a adoctrinar o manipular, sino a colocar las bases de un entendimiento básico de las grandes categorías, instituciones y procesos del fenómeno político. Desde el ámbito universitario la formación política puede contribuir a formar líderes y profesionales con fundado criterio político que incidan en el mejoramiento de la sociedad.

En el contexto de la globalización económica, la transformación de valores sociales y la pérdida de ciudadanía, la cátedra propuesta como filosofía política pretende incitar el estudio y reflexión del complejo pero vital universo de la política. Es por ello que la educación ciudadana y política se debe convertir en parte fundamental en todo programa académico que tenga por objeto los procesos de enseñanza y de aprendizaje en la educación superior. También, está más que justificada la presencia de esta asignatura en el contexto colombiano, cuyo conflicto de más de 50 ańos polariza las opciones políticas en medio de un conflicto que involucra diversos actores violentos, enmarcados todos ellos con estructuras de marginalidad, injusticia y opresión. 


\section{Reflexiones sobre la fundamentación epistemológica de la filosofía política}

Ante la necesidad de una cátedra de política en la formación profesional es fundamental diferenciar conceptos como ideologías políticas, ciencias políticas y filosofía política, para contextualizar el estudio de la política en la educación superior.

En primer lugar, las ideologías políticas tienen que ver con partidos, defensa de intereses, representación y gestión de los mismos, así como con la forma que el Estado se presenta. Es decir, no tienen una relación directa -en el sentido epistémico-con la Universidad. Las ideologías políticas, las preferencias políticas y la política como práctica no tienen nada que ver epistémicamente con la ciencia ${ }^{3}$.

Por su parte, las ciencias políticas son un saber especializado sobre la misma. Esa especialización consiste en el centramiento del politólogo en ciertos aspectos de la política que pueden ser medibles. Es así porque la ciencia trabaja sobre elementos fácticos que se traducen en hipótesis, que deben ser verificadas y medibles para obtener conocimientos seguros denominados principios o leyes. Actualmente la ciencia política se ha matematizado profundamente ${ }^{4}$. Esa matematización se logra a través de la estadística. De tal manera que lo que se llama ciencia política suele hoy estar respaldado en la estadística.

La estadística sirve para medir la opinión de los ciudadanos sobre las decisiones políticas a tomar, la conformidad o no con el gobierno de turno, la aceptación y confianza de los gobernantes, la legitimidad de un gobierno, la tendencia electoral, entre otras. Así mismo, es usada para proyectar actuaciones políticas y toma de decisiones. Es una ciencia basada primordialmente en una metodología positivista que requiere de la verificación e interpretación medibles de los hechos sociales que se estudian 5 .

De otro lado, la filosofía política pretende reflexionar sobre los conceptos de la política. Toma elementos de las ideologías políticas como de la ciencia política, para estructurar esa

3 Andrés Botero, Filosofía del derecho e historia del derecho: espacios para el encuentro (Medellín, 2001), http://goo.gl/SVzBNq

4 Paulo Ravecca, Memorias del Congreso de la ciencia politica y la politica de la ciencia: un ejercicio de introspección disciplinar desde América Latina hoy (La Habana, Cuba, 2007), http://goo.gl/NKRuaA

5 Pilar Zambrano, El derecho como práctica y como discurso. La perspectiva de la persona como garantía de objetividad y razonabilidad en la interpretación (Bogotá: Universidad de la Sabana, 2004), http://goo.gl/ jTSO4P 
reflexión conceptual. Recurre a la historia para comprender el sentido de esos conceptos en los contextos específicos que se presentaron. Su fundamento está en la filosofía misma, en su naturaleza, en su proceder, sus métodos y sus objetivos.

Se trata de pensar, reflexionar, crear y llegar a los conceptos y, entre estos, tenemos algunos como la libertad, el poder, la legitimidad, la legalidad, el buen gobierno, los derechos humanos, la democracia, los partidos políticos, el conflicto, el posconflicto, y muchos otros. Es una reflexión que toma la política como el arte de vivir en comunidad en forma pacífica y solidaria. Esto no se reduce al subjetivismo ni al capricho del analista, sino que se puede acordar, debatir, pensar entre los académicos e intelectuales que tratan sobre estos temas.

Como lo expresa Adela Cortina en su texto 10 palabras claves en Filosofía Política ${ }^{6}$, el filósofo de la política debe conocer bien las tradiciones de la filosofía política, estudiar las aportaciones científicas y, sobre todo, hacer pie en la realidad social, que es la que importa comprender.

Ahora bien, avanzando en la comprensión sobre el quehacer de la filosofía política, hay que tener en cuenta que cuando se habla de la concepción filosófica sobre la política, suele pensarse que se trata del examen de las ideas políticas que en el pasado algunos pensadores plantearon, por lo que la tarea desde el presente consistiría en analizar sus contenidos, intentar establecer su actualidad y, en el mejor de los casos, conocer sus contextos históricos. No cabe duda que tal visión sobre la filosofía de la política se inscribe, de manera general, en una concepción de la filosofía en la que se entiende la tradición filosófica, más que como un proceso abierto, como el marco ineludible desde el cual debemos pensarnos en el presente.

Frente a esta forma de comprender la filosofía política podemos, a partir de una inserción en esa tradición, indagar en el concepto mismo de política y desde ahí entender las tareas de la investigación que de ella se derivan. En primer lugar, se trata de un concepto que en su integralidad aparece como síntesis de la vida social. Al respecto, se puede afirmar que la política es el punto de articulación entre las distintas dimensiones de la vida social: lo económico, lo social, lo militar, lo ético, lo pedagógico, lo natural y lo propiamente político; esto es las formas de gobierno y su relación con la sociedad. Así entendida, la política mantiene con la ética una relación constitutiva toda vez que en su relación dinámica ellas establecen el horizonte y el sentido mismo de la vida en sociedad. Bien sea bajo la forma del bien común y la paz, de la felicidad o de la virtud, ellas definen la razón de ser de la sociabilidad humana más allá de la fundamental satisfacción de las necesidades básicas.

6 Adela Cortina, 10 palabras clave en Filosofia Politica, (Pamplona: Editorial Verbo Divino, 1998). 
El concepto de política, sin embargo, no se define solamente a partir de una relación conceptual dinámica, sino también de formas de contrastación entre los conceptos y realidades históricas: gobiernos, constituciones, confrontaciones, guerras, experiencias políticas, y aún contextos geográficos y naturales.

Si de un lado hablamos de un concepto filosófico integral de política, del otro hablamos de tres niveles a través de los cuales podemos acceder a su comprensión: los conceptos o las ideas, las relaciones y las formas. En el primer nivel, de los conceptos, encontramos los contenidos a través de los cuales comprendemos la política en una época determinada, aún los discursos que los poderes o contrapoderes producen para neutralizar al adversario o ganar una posición determinante en un escenario específico.

Al nivel de las relaciones podemos advertir los distintos modos a través de los cuales los factores reales de poder en una sociedad producen dinámicas de choque, cooperación, convivencia, aniquilamiento, entre ellos y los dispositivos, tácticas y estrategias que ponen en juego, pero también el cruce de las distintas dinámicas de la vida social que operan sobre la política o viceversa. En cuanto a las formas, se entienden como los modos y mecanismos o dispositivos a través de los cuales se organiza la voluntad política bien sea desde las dinámicas de los factores de poder que expresan la hegemonía política o aquellos que se oponen a ella.

De esta manera encontramos en la filosofía política no solo la posibilidad de comprender el carácter integral de la política, sino la existencia de los tres niveles desde los que podemos entenderla, tanto de manera general como en su existencia específica en una época determinada. Ahora bien, siguiendo a Cortina ${ }^{7}$, se pueden resumir los aspectos de la filosofía política de la siguiente manera:

- El triunfo de la democracia sobre otras formas de gobierno después del final de la guerra fría, ha develado una serie de dificultades y contradicciones que se pueden expresar en los siguientes planteamientos: ¿`el pueblo es realmente el soberano?, ¿`sus representantes están más pendientes de él o de los medios de comunicación que los rodean?, ¿el fin de la llamada cosa pública, es el pueblo o más bien los partidos políticos?, ¿`sí existe la verdadera separación de poderes para el control del Estado?, ¿el pueblo es el protagonista de la política?, ¿qué sucedió con el Estado de bienestar que había alentado la ilusión de una mayor igualdad?

- El tema de la justicia es motivo de debate en la medida en que obliga a reflexionar sobre la forma en que se debe armonizar el pluralismo social con la defensa común de

7 Ibíd. 
una Constitución democrática. El multiculturalismo plantea esa inquietud en el sentido que cada cultura pretende a la luz del derecho garantizar lo que considera son valores históricos de su pueblo; y en algunos casos es imposible garantizar ciertas prebendas en aras de la justicia o muchas veces hay que marginarse de lo que es justo para los demás por posibilitar sus expresiones.

- Las crisis del Estado de bienestar y del Estado nacional, dieron paso a las comunidades trasnacionales y a las alianzas cosmopolitas que exigen la globalización y la nueva dinámica de los mercados; los interrogantes generados tratan sobre el nuevo concepto de nación, de soberanía y de Estado. En este sentido, la comunidad internacional emerge como un actor relevante en las decisiones que se adoptan e incluso como un referente que los países han de tener presente a la hora de tomar decisiones de carácter interno.

Las dinámicas de la sociedad actual obligan al estudioso del tema a estar atento a los cambios que la sociedad política va experimentando como resultado de los avances tecnológicos, las estrategias económicas y la nueva manera de pensar y de actuar de los seres humanos. Y mantienen a la Universidad como el centro de reflexión y el lugar en el que se forman académicamente los futuros profesionales y ciudadanos.

\section{Relevancia de la reflexión sobre la ciudadanía}

El avance globalizador, los cambios sociales y la relación entre el manejo y alcance de los intereses públicos parecen distanciarse del actuar político, debilitando la institucionalidad democrática. Siendo así, es pertinente que desde los espacios académicos se trabaje para dotar a los profesionales de elementos que les permitan comprender su condición como ciudadanos y actores comprometidos con la construcción de una cultura democrática y participativa, y al liderazgo de proyectos que hagan posible una nueva sociedad.

¿Pero qué clase de ciudadano pretendemos formar? Sin duda, el término nos remite al actuar político generalizado en las polis griegas, en el que se forjaron los ideales democráticos. Su identidad, estrechamente vinculada con la comunidad, hizo que el ciudadano encontrara como razón de su existencia la acción política como forma suprema de la vida, dirigida a la construcción del orden político y que su participación resultara determinante en los destinos de 
la polis, que expresa con acierto Aristóteles al señalar que "la prosperidad de la asociación es su obra común y la asociación en este caso es el Estado"

Para llegar a estos niveles de compromiso, el mecanismo no será otro que el desarrollo de un proyecto educativo que conjugue valores cívicos y éticos haciendo del ciudadano un miembro activo en la vida del Estado y a su vez recibiendo de este los valores que contribuyen a su dignificación como persona y como ser social.

La relación ciudadano-Estado, como interacción de doble vía al interior de la comunidad, se perdió en la modernidad por el concepto abstracto y universal de ciudadanía que se propuso en la Declaración Universal de los Derechos del Hombre y del Ciudadano, que pasó a exaltar el reconocimiento de la persona como sujeto de derechos, centrándose en la individualidad y debilitando todo compromiso para actuar como miembro de una comunidad que se integra en aras del bien común.

Desde entonces, las democracias dejaron de lado la proyección social que el Estado debía cumplir y plantearon como opción voluntaria del ciudadano ser partícipe de acciones que afirmen la solidaridad e identidad como miembro de su sociedad política. Así, el ciudadano se convirtió en un sujeto pasivo, despojado de todo sentido de pertenencia, en una sociedad global e interconectada que lo aisló más del Estado, convirtiéndolo en un ser cada vez más apolítico, abstencionista y radical frente a los problemas y fenómenos políticos actuales.

Sin embargo, el enfoque liberal de ciudadanía requiere ser replanteado teniendo en cuenta que hay algunos factores que permiten ensanchar esta noción. De un lado, los movimientos sociales han contribuido a la ampliación de la comprensión de la ciudadanía como un espacio de participación alternativa a la generada por el Estado y los partidos, permitiendo la inclusión y estableciendo nuevas relaciones sociales. De otro lado, la multiplicidad discursiva, la heterogeneidad social y la diversidad cultural, son dinámicas que acompañan las dinámicas contemporáneas y complejizan el debate sobre la tensión entre homogeneidad legal vs. diversidad cultural, imperativo de igualdad vs. derecho a la diferencia, y libertad positiva vs. libertad negativa?

De esta manera la cátedra de filosofía política busca transcender cualquier concepción homogenizante y universalizante sobre la ciudadanía, ofreciendo la oportunidad de reflexionar acerca de la complejidad que implica el ejercicio ciudadano en un contexto democrático y resaltando que precisamente por tal complejidad es necesario asumirlo con responsabilidad,

8 Aristóteles, La Política, (Buenos Aíres. Austral, 1985), 106.

9 Grupo de investigación Cultura Política y Poder Constituyente, Cultura política, ciudadanía y democracia. No. 3. (Bogotá: Universidad Nacional de Colombia, 2005), 35. 
pues su incidencia permea e incide radicalmente en los diversos ámbitos humanos, tanto a nivel individual como social.

\section{La cultura política de la mano de la ética cívica}

Ante el avance globalizador, el Estado enfrenta nuevos problemas, como la pobreza en la mayoría de sus habitantes, la desigualdad social, la exclusión de amplios sectores sociales, la apatía política y la corrupción en todas sus formas, que se constituyen en ingredientes que debilitan la democracia. Es necesario por tanto, como lo seńala Robert Putnam, crear una comunidad cívica, capaz de desplegar una acción política conducente a la creación de un capital social, entendido como "el conjunto de redes, normas y confianza que posibilita a los participantes una participación conjunta y eficaz para alcanzar objetivos compartidos ${ }^{10}$.

De esta propuesta se desprende la necesidad de propiciar la integración social mediante redes de relaciones entre el Estado y la sociedad, promoviendo los valores comunitarios y ciudadanos, dado que no pueden seguir siendo una abstracción, sino una vivencia que nos permita reconocernos como parte de la comunidad, en la que se actúa por y para ella, fortaleciendo la institucionalidad del Estado y el bienestar social. Bajo estos presupuestos, es preciso que la Universidad adopte como parte de su proyecto educativo, la implementación de una cultura política que logre rescatar la idea de ciudadanía, como una forma de trascendencia del individuo en la comunidad. El significante ciudadano, al igual que el de democracia, se refieren al sujeto que se trasciende a sí mismo y se conecta con los otros en una nueva forma de existencia: la comunidad ${ }^{11}$.

De otra parte, todo intento por fortalecer la cultura política, requiere crear mecanismos de convivencia, búsqueda de consensos, respeto por las diferencias y las instituciones, y el trabajo unánime para demandar de los gobernantes y asumir como gobernados una mayor sensibilidad por los problemas sociales. La indiferencia y falta de confianza en la dirigencia política y en los gobernantes radica en el desconocimiento de los problemas y en la falta de sensibilidad para solucionar las demandas. Es en este ambiente donde se hace urgente plantear, como principal

10 Alejandra Ríos Cázares y Julio Ríos Figueroa, Capital social y democracia: una revisión crítica de Robert Putnam. Politica y Gobierno, Vol. VI N². (1999).

11 Miguel De la Torre, El ciudadano democrático. Utopía sensata de la posmodernidad educativa, Revista Electrónica de Investigación Educativa, Vol. 2 №2 (2000): 47-60. 
componente de la formación política, la construcción de una ética y unos valores mínimos que nos permitan resolver pacíficamente las diferencias.

Volvemos a Adela Cortina cuando define esa ética cívica como el "conjunto de valores y normas que comparte una sociedad moralmente pluralista, que permite a los distintos grupos no solo coexistir, no solo convivir, sino también construir la vida juntos a través de proyectos compartidos y descubrir respuestas comunes a sus desafíos"12.

Los desafíos de una sociedad que se transforma, requieren armonizar los valores individuales con los de las nuevas comunidades. Para ello, es preciso desplegar un proceso educativo que permita la concientización, participación y responsabilidad del ciudadano frente a las instituciones del Estado y superar la apatía política. Confianza, reflexión, reciprocidad, cooperación, participación y compromiso cívico serán elementos que permitirán fortalecer la democracia actual.

Finalmente, es necesario orientar el debate hacia la creación de actitudes de pertenencia en una colectividad, capaz de construir un proyecto político incluyente dentro de la complejidad de relaciones de poder político, económico, institucional, legal, ético, cultural y social, bajo la necesidad de descubrir el entorno en el que nos desenvolvemos ante la realidad del mundo globalizado.

\section{Metodología de trabajo propuesta para una cátedra de filosofía política}

En primera instancia, es necesario partir de las múltiples problemáticas que se enfrentan actualmente a la hora de trabajar la cátedra de filosofía política, en Colombia:
a. Narcotráfico
b. Violencia
c. Déficit de legitimidad
d. Gobernabilidad
e. Inserción (y exclusión)
f. Pobreza
g. Ciudadanía

12 Gabriela Giménez y Valeria Muñoz, Reseña. Cortina, Adela: "Hasta un Pueblo de Demonios" Ética Pública y Sociedad. Ed. Taurus. Pensamiento, Revista Confluencia. Vol. 1 N4 (2004), http://goo.gl/ X5h3Gf 
h. Integración nacional

i. Medio ambiente

Por supuesto, existen muchas más problemáticas, pero debemos reconocer que estas son las que tienen una mayor fuerza en cuanto al tratamiento del tema se refiere y, como dice Jaramillo Salazar, se trata de encontrar un camino explicativo e interpretativo que contribuya a revelar la esencia de la precariedad de nuestra sociedad ${ }^{13}$.

Teniendo en cuenta las definiciones iniciales, y asimilando el planteamiento de la problemática a nivel nacional, se presentan algunos aspectos problemáticos a partir de los cuales se encuentran motivos de discusión en términos epistemológicos, pedagógicos, didácticos y políticos.

En primer lugar, se debe tener en cuenta que existen presupuestos socioculturales generalizados, probablemente acompañados de referentes ideológicos imprecisos y que distorsionan la realidad, según los cuales la mayoría de los adolescentes están en capacidad de repetir ideas como:

- Todos los políticos son ladrones.

- Con la política no se come.

- La política no sirve sino para conseguir problemas.

- La política es corrupción y nada más.

- La política no es más que una mancha permanente de sangre.

- El Congreso de la República no hace nada.

Otro punto de importancia hace referencia a que el trabajo que se lleve a cabo en la cátedra de filosofía política es de una verdadera delicadeza académica, pues es necesario balancear el conocimiento entre la rigurosidad y su vulgarización. Un problema central del curso estaría relacionado con el desconocimiento de un vocabulario apropiado para el análisis político en su más mínima expresión. Aquí debe tenerse en cuenta que la mayoría de edad implica ciudadanos que tengan conceptos más o menos claros del Estado, poder, gobierno, y en general de todas aquellas categorías que constituyen el mundo de lo político. Desafortunadamente la apatía generalizada respecto a la política hace que muchas veces se tenga que detener el reloj para explicar cuestiones que se supone deberían ser conocidas de antemano.

Es necesario aclarar que, como parte integral del método de trabajo de la cátedra de filosofía política que se propone como formación política en el contexto de la formación

13 Hernán Jaramillo Salazar, ¿Científicos, educadores o rentistas? Un diálogo desde el “Almendrón”, en Hernando Gómez Buendía ¿Para dónde va Colombia? Un coloquio abierto, (Bogotá: TM editoresColciencias, 2001). 
profesional, lo primero que se debe hacer es intentar el desmonte de las percepciones erradas de la realidad. No en el sentido de que exista una verdad única, sino que debe realizarse el ejercicio del pensamiento para concluir posibles verdades, fruto de la autonomía del pensamiento y de la dialéctica de la sociedad.

La forma de lograrlo es, en un primer momento, mediante el desarrollo de procesos de sensibilización que permitan un acercamiento al mundo de lo político, utilizando elementos de las actuaciones políticas que para los estudiantes muchas veces pasan desapercibidos, pero que en el aula de clase se retoman para que generen nuevas interpretaciones, nuevas visiones, sobre el quehacer político nacional. Es necesario comprender que para alcanzar este objetivo se requieren elementos interdisciplinarios que permitan explicar por qué actuamos de ciertas maneras en el ámbito de lo político y no de otras.

Posteriormente, se recurre a la comprensión del fenómeno político a partir del estudio y el análisis de los elementos teóricos que fundamentan la filosofía política. Finalmente, los estudiantes deben apropiarse reflexivamente de la realidad política del país, con el objeto de crear ciudadanos democráticos, participativos y capaces de resolver los conflictos de su comunidad.

Estos tres aspectos se ejercitan a través de mediaciones pedagógicas tales como:

- Análisis de documentos: noticias, artículos de opinión, textos sugeridos.

- Dinámicas audiovisuales: cine foro.

- Actividades de apoyo: conferencias y debates.

- Realización de foros.

- Investigaciones de aula.

- Salidas pedagógicas.

Por este medio se intenta acompañar al estudiante, para que ingrese por los caminos de la reflexión política, con la intención de que reconozca y se apropie de un conocimiento básico sobre la organización política de la sociedad en la que vive. De esta manera se pretende contribuir a la formación integral de los profesionales, invitándolos a la construcción de una democracia participativa que conlleve a la transformación de la realidad local y nacional.

Se busca que con esta formación política los estudiantes se empoderen con el ánimo de coadyuvar a la transformación del Estado excluyente, corrupto, antidemocrático, clientelizado, ineficiente y burocrático, en otro que sea incluyente, pluralista, transparente y en el que predomine el bienestar y el interés colectivo ${ }^{14}$.

14 Miguel Eduardo Cárdenas, Modernidad y Sociedad Política en Colombia, (Bogotá: Ediciones Foro Nacional por Colombia, 1993). 


\section{Referencias}

Aristóteles. La Política. Buenos Aires: Austral, 1985.

Botero, Andrés. Filosofía del derecho e historia del derecho: espacios para el encuentro. Medellín, 2001. http:// goo.gl/SVzBNq

Cortina, Adela. 10 palabras clave en filosofía politica. Pamplona: Editorial Verbo Divino, 1998.

Cárdenas, Miguel Eduardo. Modernidad y sociedad politica en Colombia. Bogotá: Ediciones Foro Nacional por Colombia, 1993.

De la Torre, Miguel. El ciudadano democrático. Utopía sensata de la posmodernidad educativa. Revista Electrónica de Investigación Educativa Vol. 2 N² (2000): 47-60.

Giménez, Gabriela y Muñoz, Valeria. Reseña. Cortina, Adela: "Hasta un Pueblo de Demonios” Ética Pública y Sociedad. Ed. Taurus. Pensamiento. Revista Confluencia. Vol. 1 N4 (2004). http://goo.gl/ X5h3Gf

Grupo de investigación Cultura Política y Poder Constituyente. Cultura política, ciudadanía y democracia. No. 3. Bogotá: Universidad Nacional de Colombia, 2005.

Jaramillo Salazar, Hernán. ¿Científicos, Educadores o Rentistas? Un Diálogo desde el “Almendrón”. En Gómez, Buendía Hernando. ¿Para dónde va Colombia? Un coloquio abierto. Bogotá: TM editores Colciencias, 2001.

Ravecca, Paulo. Memorias del Congreso de La ciencia politica y la política de la ciencia: un ejercicio de introspección disciplinar desde América Latina hoy. La Habana, Cuba. 2007. http://goo.gl/NKRuaA

Ríos Cázares, Alejandra y Julio Ríos Figueroa, Capital social y democracia: una revisión crítica de Robert Putnam. Politica y gobierno, Vol. VI N². (1999).

Zambrano, Pilar. El derecho como práctica y como discurso. La perspectiva de la persona como garantía de objetividad y razonabilidad en la interpretación. Bogotá: Universidad de la Sabana, 2004. http://goo. $\mathrm{gl} / \mathrm{jTSO} 4 \mathrm{P}$ 